EDITORIAL

\title{
New tools for assessing microvascular obstruction in patients with ST elevation myocardial infarction
}

\author{
J A de Lemos, J J Warner
}

While early and sustained patency of the infarct related artery is necessary following ST elevation myocardial infarction, tissue and microvascular perfusion must also be restored. Effective tools for assessing microvascular obstruction are therefore essential

See end of article for authors' affiliations

Correspondence to: James A de Lemos, MD, UT Southwestern Medical Center, 5323 Harry Hines Blvd, HA 9.133, Dallas, TX 75390-9047, USA; james.delemos@ utsouthwestern.edu
A primary goal of therapy in ST elevation myocardial infarction (MI) has been to restore normal blood flow in the occluded epicardial coronary artery as rapidly as possible. The "early open artery hypothesis" is supported by large datasets demonstrating a stepwise association between faster post-reperfusion epicardial blood flow and lower subsequent mortality. ${ }^{1}$ A series of studies, using a variety of invasive and non-invasive imaging techniques, has shown that while early and sustained patency of the infarct related artery (IRA) is necessary, it is not sufficient to ensure optimal outcomes after reperfusion therapy: tissue and microvascular perfusion must also be restored.

Using myocardial contrast echocardiography (MCE), a technique that was novel at the time, Ito and colleagues demonstrated microvascular "no reflow" among $\sim 25 \%$ of patients with normal epicardial blood flow following primary percutaneous coronary intervention (PCI) for acute MI. ${ }^{2}$ These patients were found to be at high risk for subsequent death and heart failure. Using tools as diverse as intracoronary Doppler, cardiac MRI, nuclear scintigraphy, and contrast angiography, it has been shown that microvascular impairment is associated with an adverse prognosis, even if epicardial blood flow has been restored. $^{3}$

\section{ASSESSING REPERFUSION THERAPY}

A less sophisticated technique, measurement of the degree of resolution of ST elevation on the surface 12 lead ECG, has long been used to assess success or failure of reperfusion therapy. As the definition of successful reperfusion has evolved, so has understanding of the information provided by the ECG. ${ }^{4}$ When assessed 90-180 minutes after the administration of fibrinolytic treatment, complete resolution of ST elevation is associated with a very high (90-95\%) probability of a patent IRA, and an $\sim 80 \%$ probability of TIMI (thrombolysis in myocardial infarction) grade 3 flow. ${ }^{5}$ In contrast, failure to completely resolve ST elevation is not associated with a particularly high rate of an occluded IRA. At least $50 \%$ of patients with persistent ST elevation 90-180 minutes after fibrinolytic treatment have a
Heart 2004;90:119-120. doi: 10.1136/hrt.2003.018093

patent IRA at the time of early catheterisation. ${ }^{5}$ Interestingly, patients with persistent ST elevation despite restoration of normal epicardial blood flow are denied the full benefits of successful reperfusion-they remain at high risk for death and heart failure. ${ }^{6}$ Recently, studies using myocardial contrast echocardiography, ${ }^{7}$ Doppler flow wire measurements, ${ }^{8}$ angiographic perfusion grading, ${ }^{9}$ and nuclear scintigraphy ${ }^{10}$ have shown significant correlation between microvascular injury and persistent ST elevation among patients with TIMI grade 3 flow.

\section{THE INTRACORONARY PRESSURE WIRE} AS A TOOL TO DETECT MICROVASCULAR OBSTRUCTION

In this issue of Heart, Sezer and colleagues assess another tool for evaluating the coronary microcirculation after reperfusion therapy, the intracoronary pressure wire. ${ }^{11}$ They report findings from 33 patients with ST elevation MI who were treated with fibrinolytic therapy, had paired ECGs performed before and 90 minutes after treatment, and achieved TIMI grade 3 flow by the time of the angiography (mean of four days post-MI). At the time of elective stenting, the investigators used a pressure wire that also functioned as the guide wire for intracoronary stenting. Maximal hyperaemia was induced with administration of routine doses of intracoronary adenosine. When the delivery balloon was inflated during stent deployment, occluding antegrade flow, mean pressure distal to the stenosis was recorded as the coronary wedge pressure (CWP). The authors defined the pressure derived collateral flow index (CFIp) as: CWP - central venous pressure/proximal aorticcentral venous pressure. ${ }^{11}$

This index has been validated as a tool to evaluate collateral flow in non-infarct settings. In patients with stable angina pectoris undergoing elective PCI, elevations of coronary wedge pressures and CFIp $(\geqslant 0.25)$ are inversely correlated with the size of the ischaemic territory measured by nuclear SPECT (single photon emission computed tomography) imaging during balloon occlusion ${ }^{12}$ and with both acute and long term ischaemic events following PCI. ${ }^{13}{ }^{14}$ In this

Abbreviations: CAD, coronary artery disease; CFIp, pressure derived collateral flow index; CWP, coronary wedge pressure; IRA, infarct related artery; MCE, myocardial contrast echocardiography; MI, myocardial infarction; $\mathrm{PCl}$, percutaneous coronary intervention; SPECT, single photon emission computed tomography; TIMI, thrombolysis in myocardial infarction 
setting, recruitable collateral blood flow exerts a protective effect, raising CFIp and reducing ischemia.

In patients with acute MI, elevations in CWP and CFIp appear to reflect a different pathophysiology. Here, the correlation with angiographic measurements of collateral blood flow is poor, and increased CWP and CFIp appear to reflect increased "back pressure" from obstruction of the coronary microcirculation. ${ }^{15}$ This observation is predictable because plaque rupture in acute MI often involves a previously non-obstructive coronary lesion, without the well developed collateral circulation seen in patients with chronic stable angina.

In the present study, the investigators correlated CWP and CFIp with ST resolution among patients with normal epicardial blood flow following fibrinolytic treatment. ${ }^{11}$ They found an inverse correlation between CWP and ST resolution $(R=-0.64 ; \mathrm{p}<0.01)$. Furthermore, mean CWP was $55 \%$ higher in the group of patients with poor $(<50 \%)$ ST resolution than the group with good $(\geqslant 50 \%)$ ST resolution. Almost identical results were noted for CFIp. No association between CFIp and collateral grade was detected, supporting the hypothesis that in the setting of a recent acute MI, CWP and CFIp predominantly reflect the degree of microvascular obstruction. These observations are consistent with a previous study by Yamamoto and colleagues in which patients with MCE evidence of microvascular no reflow following primary PCI had higher CWP and CFIp. ${ }^{15}$ In contrast, a study by Lee and associates reported more angiographic collaterals and greater recovery of left ventricular function among patients with higher CFIp. ${ }^{16}$ This study was limited in that the investigators did not administer intracoronary vasodilators before pressure measurements. ${ }^{16}$

\section{NEXT STEPS}

These hypothesis-generating studies have laid the groundwork for broader investigation, which should concentrate on resolving many of the discrepancies generated by the existing literature. First, future studies should follow the example of Sezer and colleagues ${ }^{11}$ and measure CWP during maximal hyperaemia induced by coronary vasodilators. This is particularly important following acute MI, when epicardial and microvascular flow are dynamic. This convention will facilitate the comparison of results across the rapidly evolving literature on this topic. Second, in addition to CFIp, studies should independently report CWP as a variable. In the present study, CWP was more closely correlated with ST resolution than was CFIp. Similarly, in the initial study by Yamamoto and colleagues CWP, but not the other measured variables included in the CFIp calculation, varied significantly between those with and without evidence of microvascular obstruction. ${ }^{15}$ Central venous access is not routinely obtained during PCI in most centres, so if a simple measurement of CWP conferred similar prognostic information to CFIp, the routine practice of determining CWP would more quickly gain acceptance in the interventional community.

Other questions also remain about the role of CWP measurement in patients with ST elevation MI. These pilot studies have evaluated selected groups of patients, who are not likely to have confounding factors that might impact CWP or CFIp. For example, the present study included only patients with single vessel coronary artery disease (CAD). In patients with multi-vessel CAD, collateral blood flow may be increased, and could contribute to CWP elevation that would falsely suggest microvascular obstruction. Similarly, patients with chronic left ventricular hypertrophy or left ventricular dysfunction may have microvascular impairment that is not caused by the acute MI. Studies are needed to evaluate the validity of the CWP measurement in more heterogeneous patient populations.

Despite these limitations, the authors should be congratulated for the application of a novel measurement that may have broad implications for assessing the microcirculation in acute MI. Given its simplicity of measurement, and correlation with microvascular obstruction, CWP and/or CFIp may prove to be useful modalities for evaluating the efficacy of therapeutic strategies for microvascular protection in patients with acute coronary syndromes who undergo PCI. It is possible that treatments such as glycoprotein IIb/IIIa inhibitors, thienopyridines, and distal protection devices may all lower CWP. Patients with low CWP following PCI for acute MI may have improved outcomes and be candidates for an accelerated hospital discharge or an abbreviated course of glycoprotein II/IIIa inhibitors. If CWP and/or CFIp can be established as end points with important prognostic value, it will support the argument that routine coronary physiology measurements during PCI are worth the time, trouble, and expense.

\section{Authors' affiliations}

J A de Lemos, J J Warner, Cardiovascular Division and Department of Medicine, University of Texas Southwestern Medical Center, Dallas, Texas, USA

\section{REFERENCES}

1 Gibson CM, Murphy SM, Rizzo MJ, et al. Relationship between TIMI frame count and clinical outcomes after thrombolytic administration. Circulation 1999;99:1945-50.

2 Ito $\mathrm{H}$, Tomooka T, Sakai N, et al. Lack of myocardial perfusion immediately after successful thrombolysis: a predictor of poor recovery of left ventricular function in anterior myocardial infarction. Circulation 1992;85:1699-705.

3 Roe MT, Ohman EM, Maas AC, et al. Shifting the open-artery hypothesis downstream: the quest for optimal reperfusion. J Am Coll Cardiol 2001;37:9-18

4 de Lemos JA, Braunwald E. ST segment resolution as a tool for assessing the efficacy of reperfusion therapy. J Am Coll Cardiol 2001;38:1283-94.

5 de Lemos JA Antman EM, McCabe CH, et al. ST-segment resolution and infarct related artery patency and flow after thrombolytic therapy. Am J Cardiol 2000;85:299-304.

6 van't Hof A, Liem A, de Boer M, et al. Clinical value of 12-lead electrocardiogram after successful reperfusion therapy for acute myocardial infarction. Lancet 1997;350:615-9.

7 Santoro GM, Valenti R, Buonamici P, et al. Relation between ST-segment changes and myocardial perfusion evaluated by myocardial contrast echocardiography in patients with acute myocardial infarction treated with direct angioplasty. Am J Cardiol 1998;82:932-7.

8 Feldman $\mathrm{U}$, Coste P, Furber A, et al. Incomplete resolution of ST-segment elevation is a marker of transient microcirculatory dysfunction after stenting for acute myocardial infarction. Circulation 2003:107:2684-9.

9 van't Hof AW, Liem A, Suryapranata $H$, et al. Angiographic assessment of myocardial reperfusion in patients treated with primary angioplasty for acute myocardial infarction: myocardial blush grade. Circulation 1998;97:2302-6.

10 Kondo M, Nakano A, Saito D, et al. Assessment of "microvascular no-reflow phenomenon" using technitium- $99 \mathrm{~m}$ macroaggregated albumin scintigraphy in patients with acute myocardial infarction. $J$ Am Coll Cardiol 1998;32:898-903.

11 Sezer M, Nisanci Y, Umman B, et al. New support for clarifying the relation between ST segment resolution and microvascular function: degree of ST segment resolution correlates with the pressure derived collateral flow index. Heart 2004;90:146-50.

12 Matsuo H, Watanabe S, Kadosaki T, et al. Validation of collateral fractional flow reserve by myocardial perfusion imaging. Circulation 2002; 105: 1060-5

13 Piils NH, Bech GJ, el Gamal MI, et al. Quantification of recruitable coronary collateral blood flow in conscious humans and its potential to predict future ischemic events. J Am Coll Cardiol 1995;25:1522-8.

14 Billinger M, Kloos P, Eberli FR, et al. Physiologically assessed coronary collateral flow and adverse cardiac ischemic events: a follow-up study in 403 patients with coronary artery disease. J Am Coll Cardiol 2002;40:1545-50.

15 Yamamoto K, Ito $\mathrm{H}$, Iwakura K, et al. Pressure-derived collateral flow index as a parameter of microvascular dysfunction in acute myocardial infarction. J Am Coll Cardiol 2001:38:1383-9.

16 Lee CW, Park S-W, Cho G-Y, et al. Pressure-derived fractional collateral blood flow: a primary determinant of left ventricular recovery after reperfused acute myocardial infarction. J Am Coll Cardiol 2000;35:949-55. 